\title{
Identification of equivalent mechanical properties for unreinforced masonry walls
}

\section{Christophe Mordant*}

National Fund for Scientific Research

and

Structural and Stochastic Dynamics,

University of Liège

B52/3, Quartier Polytech,

Liège 4000, Belgique

Email: mordant.ch@gmail.com

*Corresponding author

\section{Hervé Degée}

Construction Engineering Research Group

Hasselt University

Agoralaan, Building $\mathrm{H}$

Diepenbeek 3590, Belgium

Email: herve.degee@uhasselt.be

\section{Vincent Denoël}

Structural and Stochastic Dynamics,

University of Liége

B52/3, Quartier Polytech,

Liége 4000, Belgique

Email: v.denoel@ulg.ac.be

\begin{abstract}
Transverse vibrations of beams are an interesting topic for numerous engineering fields. The equations of motion develop under various assumptions and associate a frequency equation. This paper expresses the frequency equation for two specific models, using the Timoshenko beam theory. The first model is a typical cantilever beam with an additional mass attached to the free end. The second model considers a beam partially clamped at the base and with an elastic connection of the additional mass to the free end. The relevance of the used theory is discussed and the importance of each term of the equation is studied. These models find applications in unreinforced masonry. They are indeed fitted to identify, from white noise tests, equivalent mechanical properties of unreinforced load-bearing clay masonry walls including soundproofing devices in the perspective of modelling these elements with a macro-scale approach. Discrepancies with respect to current standards are underscored.
\end{abstract}

Keywords: earthquake engineering; equivalent properties; frequency equation; Timoshenko beam theory; transverse vibrations; unreinforced masonry. 
Reference to this paper should be made as follows: Mordant, C., Degée, H. and Denoël, V. (201X) 'Identification of equivalent mechanical properties for unreinforced masonry walls', Int. J. Masonry Research and Innovation, Vol. XX, No. YY, pp.ZZZ-ZZZ.

Biographical notes: Christophe Mordant has been graduated as $\mathrm{PhD}$ in Civil Engineering at the University of Liège and at the Hasselt University in 2016.

Hervé Degée is a Professor of Structural Engineering at Hasselt University and Head of the Construction Engineering Research Group. He is also an invited lecturer at the Universities of Ghent and Liege, where he got his $\mathrm{PhD}$ and used to work as a researcher for many years. His main research field is the design and analysis of structures with a specific emphasise on the global behaviour of structures combining different materials, as well as earthquake engineering. He is an active member of various standardisation commissions at the Belgian and European level.

From 2001 to 2009, Vincent Denoël was a Researcher for the National Research Fund in Belgium. He is now a Professor in Structural Engineering at the University of Liège. His areas of research concern are the random dynamics of low-dimensional systems, structural dynamics, the characterisation of dynamic actions and the numerical simulation of flexible structures and biomechanics. He teaches structural dynamics, structural design against exceptional loads, probabilities and stochastic mechanics, and perturbation methods for engineers. Vincent Denoël is also appointed as an Adjunct Professor and is a member of the PhD Doctoral School at the University of Minnesota, where he cosupervises doctoral theses. He has collaborations with important research and industrial partners in Europe and America. He owns a world record for the longest deathride installed in urban area (2010) and received the 'Junior Award' of the International Association for Wind Engineering (2015).

\section{Introduction}

Transverse vibrations of beams have been extensively studied for a few centuries. This interest has led to a huge number of research works. The problem is still investigated today given its large range of applications and for two other reasons. On the one hand, several assumptions may be formulated to project the most general equations of the theory of elasticity onto beam, rod or elastica models (Timoshenko and Goodier, 1987). Among the available beam theories, the well-known model proposed by Bernoulli and Euler relies on the importance of the bending effect and results in acceptable approximations for a number of engineering problems. Nevertheless, the model is inaccurate for the higher modes and non-slender beams. Improvements were then introduced by Lord Rayleigh (1877), Strutt (1877) and with the 'shear' model, through the integration of the rotary inertia and the shear distortion effects of the cross section, respectively. Finally, Timoshenko $(1921,1922)$ proposed a beam theory combining all these effects and giving a more adequate solution to the problems of higher modes and non-slender beams. On the other hand, the solution of these equations depends on the boundary conditions. The most classical ones are the 'freefree', 'clamped-clamped', 'hinged-hinged' or 'clamped-free' ends, but other possibilities exist, as for instance a partially clamped end. There are therefore as many solutions as combinations of theories and boundary conditions.

Solving the differential equations of motion analytically requires the development of the so-called frequency equation. Han et al. (1999) detailed these developments for the 
four above-mentioned models and for the classical boundary conditions. Other authors studied this problem with some specific details in the view of practical applications. Low $(1999,2000)$ focused on the frequency analysis of an Euler-Bernoulli beam bearing a concentrated mass at an arbitrary location and developed a modified Dunkerley formula to approximate the exact solution with shorter computational time. Because of its increasing use as robot arms, machines or structures, Bruch and Mitchell (1987), Oguamanam (2003), Salarieh and Ghorashi (2006) and Ansari et al. (2011) investigated the free vibrations of an Euler-Bernoulli or a Timoshenko cantilever beam with a rigid tip in the perspective of studying the behaviour of a flexible member. More recent contributions dealt with non-homogeneous or cracked beams (Li et al., 2013a; Khaji et al., 2009). The influence of the support conditions was also considered, as for example Timoshenko beams on Pasternak foundations (Calio and Greco, 2012) and beams on elastic end support ( $\mathrm{Li}$, 2013). A particular attention to forced vibrations of Timoshenko beams was given by Majkut (2009).

Beside the study of transversally vibrating beams, a similar theoretical approach has been used to examine the stability of beams. Several research programs have been carried out over the past 20 years (Farchaly and Shebl, 1995; Esmailzadeh and Ohadi, 2000), with some focused on shear beams (Zhang et al., 2013), investigating the consequences of variable sections (Naguleswaran, 2003) or of an elastically restrained base (HernándezUrrea and Dario Aristizábal-Ochoa, 2008).

The present paper aims at adding another contribution to the study of transverse vibrations of beams by determining the frequency equation of two particular cantilever beam models, using the Timoshenko beam theory. This paper also presents a new application of the frequency equations for the identification of equivalent mechanical properties of unreinforced masonry (URM) structural elements. Current procedures for the assessment of these properties are based on empirical relations between the properties of the masonry units and joints. New building systems have been however developed for energy efficiency coupled with economical reasons and still need to be characterised (da Porto et al., 2009). Defining these properties for the masonry structural elements is a primary requirement given that the full URM structures are usually modelled at the macro-scale with the equivalent frame method (Penna et al., 2013). The use of such an approach is widespread and provides relevant results with an interesting computational time (Lagomarsino et al., 2013; Braga and Liberatore, 1990; Kappos et al., 2002).

In this perspective, the boundary conditions of simple cantilever beams are here updated in order to reproduce experimental configurations of specimens tested in the framework of the European FP7 project SERIES (Mordant et al., 2014). The specimens are four simple load-bearing clay URM walls with thin-layer glued bed joints and a tongueand-groove system as head joints. They have two different aspect ratios, with a length of $2.10 \mathrm{~m}$ (corresponding to the 'long walls' here under) and $0.72 \mathrm{~m}$ (corresponding to the 'short walls' here under), respectively. An additional 5-ton steel mass is placed on the walls and one wall of each aspect ratio includes soundproofing rubber layers located at the base of the wall and between the wall and the additional mass (Figure 1). These specimens have been tested on a shake table with a characterisation of their dynamic properties prior to each shake. The geometry of the tested specimens involves the use of the Timoshenko beam theory due to the importance of the shear deformability. Interested readers can refer to Mordant (2016) for details about the specimens and the experimental campaign.

A first model is focused on walls without soundproofing devices and corresponds to the classical cantilever beam with an additional mass located at the free end (Figure 2b). 
A second model considers the walls with rubber layers and has to particularities due to these latter:

$\mathrm{i}$ the base end is elastically restrained

ii the additional mass is not rigidly connected to the free end (Figure 2c).

Figure 1 Rubber devices used for acoustic performances (see online version for colours)

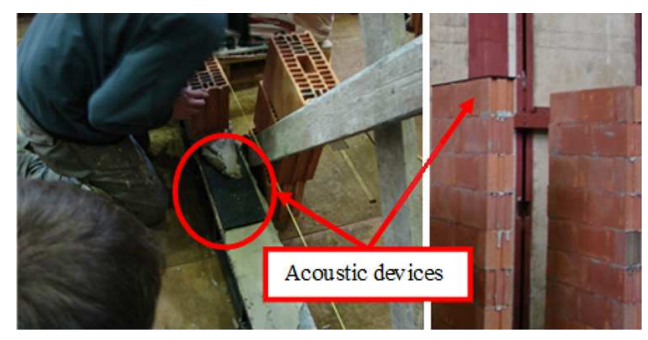

Figure 2 General sketches of (a) a cantilever beam including an additional mass and flexible layers, (b) model 1 and (c) model 2 (see online version for colours)

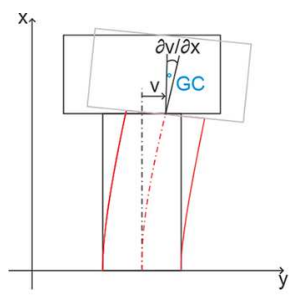

(a)

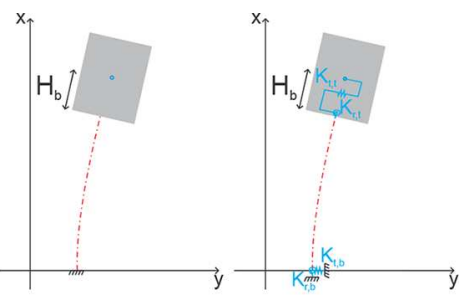

(b) (c)

Both translational and rotational flexibilities are considered. For both models, the influence of the additional mass is studied through

i its size (i.e. its geometry)

ii its relative magnitude referred to the mass of the wall

iii its rotary and translational inertia

iv the distance between its centroid and the beam free end (i.e. the top of the wall).

In particular, the novelty of the second model is to consider a flexible connection between the free end and the additional mass.

Once the dimensionless frequency equations are obtained, they are used to identify equivalent mechanical properties of the experimental specimens. This paper being focused on the identification of the dynamic properties and not the seismic response, only experimental results in terms of natural frequencies are useful (Mordant, 2016). During this phase, the walls behave in the elastic range, since they are submitted to a very low excitation. Then, the importance of the different terms of the frequency equations is investigated. Finally, equivalent mechanical properties of the tested specimens are identified and the results are compared to current standard recommendations. 


\section{Model description using Timoshenko beam theory}

\subsection{Equations of motion}

The Timoshenko beam theory includes bending, shear distortion and rotary inertia effects. Two space- and time-dependent fields describe the behaviour of the beam, namely the transverse displacement $v^{*}\left(x^{*}, t^{*}\right)$ and the angle of rotation due to bending $\alpha\left(x^{*}, t^{*}\right)$. Two governing differential equations are derived from Hamilton's variational principle

$$
\begin{aligned}
& \rho^{*} A^{*} \frac{\partial^{2} v^{*}}{\partial t^{*^{2}}}-k^{\prime} G^{*} A^{*}\left(\frac{\partial^{2} v^{*}}{\partial x^{*^{2}}}-\frac{\partial \alpha}{\partial x^{*}}\right)=f\left(x^{*}, t^{*}\right) \\
& \rho^{*} I^{*} \frac{\partial^{2} \alpha}{\partial t^{*^{2}}}-E I^{*} \frac{\partial^{2} \alpha}{\partial x^{*^{2}}}-k^{\prime} G^{*} A^{*}\left(\frac{\partial v^{*}}{\partial x^{*}}-\alpha\right)=0
\end{aligned}
$$

where $\rho^{*}$ is the density of the beam, $A^{*}$ and $I^{*}$ are its cross-sectional area and bending inertia, $k^{\prime}$ is a shape factor, $E$ and $G^{*}$ represent the elastic and shear moduli of the beam, respectively, and $f\left(x^{*}, t^{*}\right)$ is the transverse applied force per unit length. The used nomenclature is taken from Han et al. (1999). This set of equations can be made nondimensional by introducing

$$
x_{r e f}^{*}=H_{w} \quad \text { and } \quad t_{r e f}^{*}=\frac{1}{\omega_{1}^{*}}=H_{w}^{2} \sqrt{\frac{\rho^{*} A^{*}}{E I^{*}}}
$$

as a reference length (the length of the beam, i.e. the height of the wall in the following application) and time (the travelling time of a bending wave along the beam), respectively. The space and time parameters are therefore normalised as:

$$
x=\frac{x^{*}}{x_{r e f}^{*}} \quad ; \quad t=\frac{t^{*}}{t_{r e f}^{*}},
$$

while the transverse displacement of the beam is scaled as $v=\frac{v^{*}}{x_{r e f}^{*}}$. Assuming no transverse force per unit length and a linear elastic, isotropic and homogeneous beam with a constant cross section, the equations of motion (1) and (2) can be decoupled. With the dimensionless coordinates, they read

$$
\begin{aligned}
& \frac{\partial^{4} v}{\partial x^{4}}-\Omega_{a} \frac{\partial^{4} v}{\partial x^{2} t^{2}}+\frac{\partial^{2} v}{\partial t^{2}}+\Omega_{b}^{2} \frac{\partial^{4} v}{\partial t^{4}}=0 \\
& \frac{\partial^{4} \alpha}{\partial x^{4}}-\Omega_{a} \frac{\partial^{4} \alpha}{\partial x^{2} t^{2}}+\frac{\partial^{2} \alpha}{\partial t^{2}}+\Omega_{b}^{2} \frac{\partial^{4} \alpha}{\partial t^{4}}=0
\end{aligned}
$$

with the dimensionless groups

$$
\Omega_{a}=\frac{I^{*}}{A^{*} H_{w}^{* 2}}\left(1+\frac{E}{k^{\prime} G^{*}}\right) \quad ; \quad \Omega_{b}^{2}=\left(\frac{I^{*}}{A^{*} H_{w}^{* 2}}\right)^{2} \frac{E}{k^{\prime} G^{*}} .
$$

As the form of both differential Eqs. (5) and (6) is the same, the topology of the unknown fields are also the same. These latter can be expressed in the frequency domain 
by setting $v(x, t)=\phi(x) e^{j \omega t}$ and $\alpha(x, t)=\psi(x) e^{j \omega t}$, with $\omega=\frac{\omega^{*}}{\omega_{1}^{*}}$ the dimensionless circular frequency and $j$ the unit complex number. This translates the time synchronisation of the transverse displacement and of the angle of rotation due to bending (Han et al., 1999; Majkut, 2009). Plugging this ansatz into Eqs. (5) and (6) yields

$$
\begin{aligned}
& {\left[\phi^{\prime \prime \prime \prime}+\Omega_{a} \omega^{2} \phi^{\prime \prime}-\omega^{2} \phi+\Omega_{b}^{2} \omega^{4} \phi\right] e^{j \omega t}=0} \\
& {\left[\psi^{\prime \prime \prime \prime \prime}+\Omega_{a} \omega^{2} \psi^{\prime \prime}-\omega^{2} \psi+\Omega_{b}^{2} \omega^{4} \psi\right] e^{j \omega t}=0}
\end{aligned}
$$

where the prime symbol indicates derivatives with respect to $x$. These fourth-order homogeneous result in the general solution for the mode shapes $\phi(x)$ and $\psi(x)$

$$
\begin{aligned}
& \phi=K_{1} e^{z_{1} x}+K_{2} e^{z_{2} x}+K_{3} e^{z_{3} x}+K_{4} e^{z_{4} x} \\
& \psi=Q_{1} e^{z_{1} x}+Q_{2} e^{z_{2} x}+Q_{3} e^{z_{3} x}+Q_{4} e^{z_{4} x}
\end{aligned}
$$

where

$$
\begin{gathered}
z_{1,2}= \pm \sqrt{-\frac{\Omega_{a}}{2} \omega^{2}-\sqrt{\left(\frac{\Omega_{a}^{2}}{4}-\Omega_{b}^{2}\right) \omega^{4}+\omega^{2}}}=\mp a \\
z_{3,4}=\sqrt{ \pm\left[\frac{\Omega_{a}}{2} \omega^{2}-\sqrt{\left(\frac{\Omega_{a}^{2}}{4}-\Omega_{b}^{2}\right) \omega^{4}+\omega^{2}}\right]}=\left\{\begin{array}{l}
b \\
\tilde{b}
\end{array}\right.
\end{gathered}
$$

are the roots of the characteristic polynomial. The variables $a, b$ and $\tilde{b}$ are the dimensionless wave numbers.

Finally, Eqs. (10) and (11) can be turned into trigonometric or hyperbolic expressions. Two pairs of solutions are derived from (10) and (11) since $z_{3}$ and $z_{4}$ are either real or imaginary, depending on the circular frequency $\omega$ with respect to the critical frequency $\omega_{c}=\Omega_{b}^{-1}$. When the circular frequency is smaller than the critical frequency $\left(\omega \Omega_{b} \leq 1\right)$, the spatial (wave) solutions are expressed by:

$$
\begin{aligned}
& \phi=C_{1} \sin a x+C_{2} \cos a x+C_{3} \sinh b x+C_{4} \cosh b x, \\
& \psi=D_{1} \sin a x+D_{2} \cos a x+D_{3} \sinh b x+D_{4} \cosh b x .
\end{aligned}
$$

On the contrary, when the angular frequency is larger than the critical frequency $\left(\omega \Omega_{b} \geq 1\right)$, the solutions read

$$
\begin{aligned}
& \phi=\tilde{C}_{1} \sin a x+\tilde{C}_{2} \cos a x+\tilde{C}_{3} \sin \tilde{b} x+\tilde{C}_{4} \cos \tilde{b} x, \\
& \psi=\tilde{D}_{1} \sin a x+\tilde{D}_{2} \cos a x+\tilde{D}_{3} \sin \tilde{b} x+\tilde{D}_{4} \cos \tilde{b} x .
\end{aligned}
$$

The parameters $C_{1}, C_{2}, C_{3}, C_{4}$ and $D_{1}, D_{2}, D_{3}, D_{4}$ (resp. $\tilde{C}_{1}, \cdots, \tilde{C}_{4}$ and $\left.\tilde{D}_{1}, \cdots, \tilde{D}_{4}\right)$ in Eqs. (14) and (15) (resp. (16) and (17)) are eight coefficients to be determined to satisfy 
the boundary conditions. These coefficients can however be reduced to four as explained by Han et al. (1999), observing that they are related by

$$
\begin{aligned}
& D_{1}=-\varphi_{a} C_{2} D_{2}=\varphi_{a} C_{1} \\
& D_{3}=\varphi_{b} C_{4} D_{4}=\varphi_{b} C_{3}
\end{aligned}
$$

and

$$
\begin{aligned}
& \tilde{D}_{1}=-\tilde{\varphi}_{a} \tilde{C}_{2} \tilde{D}_{2}=\tilde{\varphi}_{a} \tilde{C}_{1} \\
& \tilde{D}_{3}=-\tilde{\varphi}_{b} \tilde{C}_{4} \tilde{D}_{4}=\tilde{\varphi}_{b} \tilde{C}_{3}
\end{aligned}
$$

with $\varphi_{a}=\frac{a^{2}+\gamma^{2} b^{2}}{\left(1+\gamma^{2}\right) a}, \quad \varphi_{b}=\frac{b^{2}+\gamma^{2} a^{2}}{\left(1+\gamma^{2}\right) b}, \quad \tilde{\varphi}_{a}=\frac{a^{2}-\gamma^{2} b^{2}}{\left(1+\gamma^{2}\right) a}, \quad \tilde{\varphi}_{b}=\frac{b^{2}-\gamma^{2} b a^{2}}{\left(1+\gamma^{2}\right) b}$ and $\gamma=\frac{E}{k^{\prime} G^{*}}=$ $\frac{2(1+\nu)}{k^{\prime}}$.

Once the boundary conditions specified, this yields four relations between the four unknown coefficients $C_{1}, \cdots, C_{4}\left(\tilde{C}_{1}, \cdots, \tilde{C}_{4}\right.$, respectively, if $\left.\omega \Omega_{b} \geq 1\right)$ which can be expressed as a matrix. The frequency equation is then obtained by setting the determinant of this matrix to zero, avoiding the trivial solution corresponding to zero coefficients, and revealing therefore the natural frequencies of the considered problem.

\subsection{Models and boundary conditions}

Two models of a cantilever beam are considered herein with specific details. The first model has an additional mass rigidly connected to the free end of the beam, as shown in Figure $2 b$. The second model is sketched in Figure $2 c$ and differs from the first one due to a partially clamped bottom end and to an elastic connection of the additional mass to the free end. In the applications considered below, these peculiarities are the consequences of flexible elements located at the beam ends and modelled by rotational and translational springs. In both models, the additional mass weighs $m^{*}[\mathrm{~kg}]$ and possesses a rotary inertia $I_{\text {cin }}^{*}\left[\mathrm{~kg} \cdot \mathrm{m}^{2}\right]$. Moreover, the centroid of the mass is at a distance $H_{b}^{*}[\mathrm{~m}]$ from the tip end of the beam. The springs defining the partially clamped end are characterised by a stiffness in rotation $K_{r, b}^{*}$ and in translation $K_{t, b}^{*}$, while $K_{r, t}^{*}$ and $K_{t, t}^{*}$ are the stiffness in rotation and in translation of the springs representing the flexible connection of the additional mass to the free end. These parameters are scaled in the same manner as above, leading to the following dimensionless quantities $m=\frac{m^{*} \omega_{1}^{*} H_{w}^{3}}{E I^{*}}, I_{c i n}=\frac{I_{c i n}^{*} \omega_{1}^{* 2} H_{w}}{E I^{*}}, H_{b}=\frac{H_{b}^{*}}{H_{w}}, K_{r, b}=K_{r, b}^{*} \frac{H_{w}^{3}}{E I^{*}}$, $K_{t, b}=K_{t, b}^{*} \frac{H_{w}}{E I^{*}}, K_{r, t}=K_{r, t}^{*} \frac{H_{w}^{3}}{E I^{*}}$ and $K_{t, t}=K_{t, t}^{*} \frac{H_{w}}{E I^{*}}$.

Details of the beam top with the additional mass are depicted in Figure 3, showing the contributions of the bending (blue) and shear (red) effects in terms of angle of rotations. The usual simplification for the shear deformation is given in Figure 3b. Under bending and shear effects, the centroid of the additional mass is subjected to a rotation $\alpha$ with respect to the $x$-axis. The angle $\gamma$, representing the shear effects, only impacts the variation of the transverse displacement along the height. This observation will be useful for the formulations of the bending moment and shear force.

To be consistent with the Timoshenko beam theory described above, a homogeneous beam with a linear and indefinitely elastic material and a doubly symmetric cross section is assumed. The consequences of the specific details are practically translated by modifying 
Figure 3 Details of the model free end (see online version for colours)

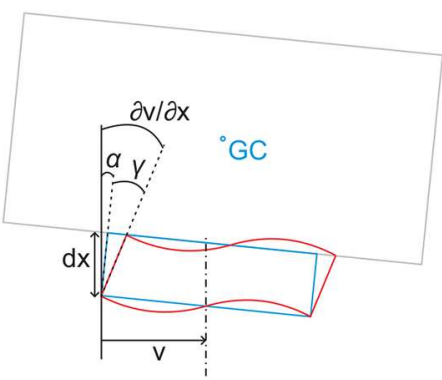

(a)

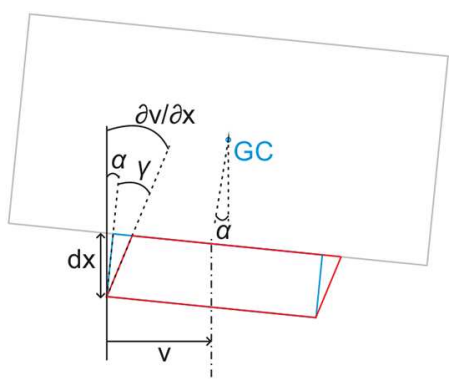

(b)

the boundary conditions of the classical cantilever beam. These latter are here expressed in a dimensionless form for the clamped $(x=0)$ and free $(x=1)$ ends, respectively,

$$
\begin{aligned}
v=0 ; \quad ; & =0 \text { at } x=0 \\
M=-\alpha^{\prime}=0 \quad ; \quad V=k^{\prime} G A\left(v^{\prime}-\alpha\right) & =0 \text { at } x=1
\end{aligned}
$$

with $G=G^{*} H_{w}^{4} / E I^{*}, \quad A=A^{*} / H_{w}^{2}$ and $M(x, t)$ and $V(x, t)$ representing the dimensionless bending moment and shear force, defined as

$$
M=\frac{M^{*}}{M_{r e f}^{*}} \quad \text { and } \quad V=\frac{V^{*}}{V_{r e f}^{*}}
$$

where $M^{*}\left(x^{*}, t^{*}\right)$ and $V^{*}\left(x^{*}, t^{*}\right)$ are the bending moment and the shear force and

$$
M_{r e f}^{*}=\frac{E I^{*}}{H_{w}} \quad \text { and } \quad V_{r e f}^{*}=\frac{E I^{*}}{H_{w}^{2}}
$$

\subsubsection{First model}

In the first model, the additional mass only influences the conditions at the free end $(x=1)$, involving non-zero bending moment and shear force

$$
\begin{aligned}
& M=-\alpha^{\prime}=m H_{b}\left(\ddot{v}+H_{b} \ddot{\alpha}\right)+I_{c i n} \ddot{\alpha} \\
& V=k^{\prime} G A\left(v^{\prime}-\alpha\right)=-m\left[\ddot{v}+H_{b} \ddot{\alpha}-H_{b} \ddot{v}^{\prime}-H_{b}^{2} \ddot{\alpha}^{\prime}\right]+I_{c i n} \ddot{\alpha}^{\prime}
\end{aligned}
$$

The magnitude $m$ and inertia $I_{c i n}$ of the mass as well as the distance $H_{b}$ are identified as the three main parameters characterising the influence of the additional mass. The prime and dot symbol indicate derivatives with respect to $x$ and $t$, respectively. 


\subsubsection{Second model}

In addition to the mass, the presence of flexible devices in the second model affects the boundary conditions at both ends. On the one hand, the beam base $(x=0)$ cannot be considered as clamped anymore. A rotation and a transverse displacement are allowed and their magnitude depends on the stiffness of the springs

$$
v=\frac{V}{K_{t, b}}=\frac{k^{\prime} G A}{K_{t, b}}\left(v^{\prime}-\alpha\right) ; \alpha=-\frac{M}{K_{r, b}}=\frac{\alpha^{\prime}}{K_{r, b}} .
$$

On the other hand, a relative rotation $\triangle \alpha$ and a relative displacement $\triangle v$ are possible between the top of the beam and the base of the additional mass due to the elastic connection. These contributions have to be taken into account and lead to the boundary conditions at the free end $(x=1)$

$$
\begin{aligned}
M= & -\alpha^{\prime} \\
= & m H_{b}\left[\ddot{v}+\ddot{\triangle} v+H_{b}(\ddot{\alpha}+\triangle \ddot{\alpha})\right]+I_{c i n}(\ddot{\alpha}+\triangle \ddot{\alpha}) \\
= & m H_{b}\left(\ddot{v}+H_{b} \ddot{\alpha}\right)+m H_{b}\left[\frac{k^{\prime} G A}{K_{t, t}}\left(\ddot{v}^{\prime}-\ddot{\alpha}\right)+\frac{H_{b} \ddot{\alpha}^{\prime}}{K_{r, t}}\right]+I_{c i n}\left(\ddot{\alpha}+\frac{\ddot{\alpha}^{\prime}}{K_{r, t}}\right) \\
V= & k^{\prime} G A\left(v^{\prime}-\alpha\right) \\
= & -m\left[\ddot{v}+\triangle \ddot{v}+H_{b}(\ddot{\alpha}+\triangle \ddot{\alpha})\right]+m H_{b}\left(\ddot{v}^{\prime}+\triangle \ddot{v}^{\prime}+H_{b} \ddot{\alpha}^{\prime}+H_{b} \triangle \ddot{\alpha}^{\prime}\right) \\
& +I_{c i n}\left(\ddot{\alpha}^{\prime}+\triangle \ddot{\alpha}^{\prime}\right) \\
= & -m\left(\ddot{v}+H_{b} \ddot{\alpha}\right)-m\left[\frac{k^{\prime} G A}{K_{t, t}}\left(\ddot{v}^{\prime}-\ddot{\alpha}\right)+\frac{H_{b} \ddot{\alpha}^{\prime}}{K_{r, t}}\right]+m H_{b}\left(\ddot{v}^{\prime}+H_{b} \ddot{\alpha}^{\prime}\right) \\
& +m H_{b}\left[\frac{k^{\prime} G A}{K_{t, t}}\left(\ddot{v}^{\prime \prime}-\ddot{\alpha}^{\prime}\right)+\frac{H_{b} \ddot{\alpha}^{\prime \prime}}{K_{r, t}}\right]+I_{c i n}\left(\ddot{\alpha}^{\prime}+\frac{\ddot{\alpha}^{\prime \prime}}{K_{r, t}}\right)
\end{aligned}
$$

Equations (24)-(26) neglect the second-order effects and the influence of the gravity is hence not considered.

\section{Frequency equations}

The frequency equations are established for the case of a circular frequency lower than the critical frequency $\left(\omega \Omega_{b} \leq 1\right)$. The equations for a circular frequency larger than the critical one can be obtained by replacing $b$ with $j \tilde{b}$.

\subsection{Frequency equation of the first model}

Equations (20) and (24) provide some relations between the four unknown coefficients, see (14). These relations are written in a matrix form as:

$$
\mathbf{A c}:=\left[\begin{array}{cccc}
0 & 1 & 0 & 1 \\
\varphi_{a} & 0 & \varphi_{b} & 0 \\
A_{31} & A_{32} & A_{33} & A_{34} \\
A_{41} & A_{42} & A_{43} & A_{44}
\end{array}\right]\left\{\begin{array}{l}
C_{1} \\
C_{2} \\
C_{3} \\
C_{4}
\end{array}\right\}=\mathbf{0}
$$


where $\mathbf{c}$ is the vector gathering the integration constants of the mode shapes $\phi(x)$ and $\psi(x)$. In matrix $\mathbf{A}$, we have introduced

$$
\begin{aligned}
& A_{31}=\left(a \varphi_{a}+\bar{P}\right) \sin a+\varphi_{a} \bar{Q} \cos a \\
& A_{32}=\left(a \varphi_{a}+\bar{P}\right) \cos a-\varphi_{a} \bar{Q} \sin a \\
& A_{33}=-\left(b \varphi_{b}-\bar{P}\right) \sinh b+\varphi_{b} \bar{Q} \cosh b \\
& A_{34}=-\left(b \varphi_{b}-\bar{P}\right) \cosh b+\varphi_{b} \bar{Q} \sinh b \\
& A_{41}=\left(a-\varphi_{a}\right)\left(1+\frac{\bar{P}}{k^{\prime} G A}\right) \cos a-\left(\frac{\omega^{2} m}{k^{\prime} G A}+\frac{a \varphi_{a}}{k^{\prime} G A}\right) \sin a \\
& A_{42}=-\left(a-\varphi_{a}\right)\left(1+\frac{\bar{P}}{k^{\prime} G A}\right) \sin a-\left(\frac{\omega^{2} m}{k^{\prime} G A}+\frac{a \varphi_{a}}{k^{\prime} G A} \bar{Q}\right) \cos a \\
& A_{43}=\left(b-\varphi_{b}\right)\left(1+\frac{\bar{P}}{k^{\prime} G A}\right) \cosh b-\left(\frac{\omega^{2} m}{k^{\prime} G A}-\frac{b \varphi_{b}}{k^{\prime} G A}\right) \sinh b \\
& A_{44}=\left(b-\varphi_{b}\right)\left(1+\frac{\bar{P}}{k^{\prime} G A}\right) \sinh b-\left(\frac{\omega^{2} m}{k^{\prime} G A}-\frac{b \varphi_{b}}{k^{\prime} G A} \bar{Q}\right) \cosh b
\end{aligned}
$$

where

$$
\bar{Q}=\omega^{2}\left(m H_{b}^{2}+I_{c i n}\right) ; \quad \bar{P}=\omega^{2} m H_{b}
$$

Setting the determinant of the matrix $\mathbf{A}$ to zero leads to the frequency equation. This equation needs to be solved for $\omega$ which lies in $a, b, \bar{Q}$ and $\bar{P}$.

\subsection{Frequency equation of the second model}

In the second model, based on Eqs. (25) and (26), the frequency equation reads

$$
\mathbf{A c}:=\left[\begin{array}{cccc}
\hat{A}_{11} & 1 & \hat{A}_{13} & 1 \\
\varphi_{a} & \hat{A}_{22} & \varphi_{b} & \hat{A}_{24} \\
A_{31} & \hat{A}_{32} & \hat{A}_{33} & \hat{A}_{34} \\
\hat{A}_{41} & \hat{A}_{42} & \hat{A}_{43} & \hat{A}_{44}
\end{array}\right]\left\{\begin{array}{l}
C_{1} \\
C_{2} \\
C_{3} \\
C_{4}
\end{array}\right\}=\mathbf{0}
$$

where

$$
\begin{aligned}
& \hat{A}_{11}=-\frac{k^{\prime} G A}{K_{t, b}}\left(a-\varphi_{a}\right) \\
& \hat{A}_{13}=-\frac{k^{\prime} G A}{K_{t, b}}\left(b-\varphi_{b}\right) \\
& \hat{A}_{22}=\frac{a \varphi_{a}}{K_{r, b}} \\
& \hat{A}_{24}=-\frac{b \varphi_{b}}{K_{r, b}} \\
& \hat{A}_{31}=A_{31}-\frac{a \varphi_{a}}{K_{r, t}} \bar{Q} \sin a+\frac{k^{\prime} G A}{K_{t, t}}\left(a-\varphi_{a}\right) \bar{P} \cos a
\end{aligned}
$$




$$
\begin{aligned}
& \hat{A}_{32}=A_{32}-\frac{a \varphi_{a}}{K_{r, t}} \bar{Q} \cos a-\frac{k^{\prime} G A}{K_{t, t}}\left(a-\varphi_{a}\right) \bar{P} \sin a \\
& \hat{A}_{33}=A_{33}+\frac{b \varphi_{b}}{K_{r, t}} \bar{Q} \sinh b+\frac{k^{\prime} G A}{K_{t, t}}\left(b-\varphi_{b}\right) \bar{P} \cosh b \\
& \hat{A}_{34}=A_{34}+\frac{b \varphi_{b}}{K_{r, t}} \bar{Q} \cosh b+\frac{k^{\prime} G A}{K_{t, t}}\left(b-\varphi_{b}\right) \bar{P} \sinh b \\
& \hat{A}_{41}=A_{41}-\frac{a \varphi_{a}}{k^{\prime} G A K_{r, t}}[a \bar{Q} \cos a-\bar{P} \sin a]-\frac{\left(a-\varphi_{a}\right)}{K_{t, t}}\left[\omega^{2} m \cos a+a \bar{P} \sin a\right] \\
& \hat{A}_{42}=A_{42}+\frac{a \varphi_{a}}{k^{\prime} G A K_{r, t}}[a \bar{Q} \sin a+\bar{P} \cos a]+\frac{\left(a-\varphi_{a}\right)}{K_{t, t}}\left[\omega^{2} m \sin a-a \bar{P} \cos a\right] \\
& \hat{A}_{43}=A_{43}+\frac{b \varphi_{b}}{k^{\prime} G A K_{r, t}}[b \bar{Q} \cosh b-\bar{P} \sinh b]+\frac{\left(b-\varphi_{b}\right)}{K_{t, t}}\left[\omega^{2} m \cosh b+b \bar{P} \sinh b\right] \\
& \hat{A}_{44}=A_{44}+\frac{b \varphi_{b}}{k^{\prime} G A K_{r, t}}[b \bar{Q} \sinh b-\bar{P} \cosh b]+\frac{\left(b-\varphi_{b}\right)}{K_{t, t}}\left[\omega^{2} m \sinh b+b \bar{P} \cosh b\right]
\end{aligned}
$$

It can be noticed that this second case degenerates into the first model if the stiffnesses $K_{t, t}, K_{r, t}, K_{t, b}, K_{r, b}$ tend to infinity.

\section{Parametric study of the beam models, frequency equations and mode shapes}

The coefficients of the matrix $\mathbf{A}$, leading to the expression of the frequency equation, highlight the importance of the geometrical and mechanical properties of the beam through the dimensionless groups $\Omega_{a}$ and $\Omega_{b}$. Additional parameters also influence this equation due to the presence of an additional mass at the top, such as the mass $m$ and its rotary inertia $I_{c i n}$ as well as the distance $H_{b}$ from the beam free end to the centroid of the mass. The second model has four extra parameters. These are the stiffnesses in rotation and in translation of the material placed at the beam ends, namely $\left(K_{r, b}, K_{t, b}\right)$ and $\left(K_{r, t}, K_{t, t}\right)$ for the base and top ends, respectively. In comparison with the classical cantilever beam, those parameters obviously add complexity in the frequency equation.

\subsection{Comparison of the Euler-Bernoulli and Timoshenko beam models}

Equations (5) and (6) express the decoupled equations of motion according to the Timoshenko beam theory. When $\Omega_{a} \ll 1$ and $\Omega_{b} \ll 1$, these equations regularly degenerate into the equation of motion given by the Euler-Bernoulli (E-B) theory. This section aims at studying the evolution of the wave numbers $a$ and $b$ (or $\tilde{b}$ ) as a function of the dimensionless groups $\Omega_{a}$ and $\Omega_{b}$. The variations of these latter are correlated in the light of the expressions of the wave numbers given in (12) and (13). Indeed, the radicand of these equations has to be positive, leading to the condition

$$
\Omega_{b}^{2}-\frac{\Omega_{a}^{2}}{4} \leq \frac{1}{\omega^{2}}
$$

Figure 4 gives a mapping of the wave numbers $a$ and $b$ (or $\tilde{b}$ ), respectively, for a dimensionless circular frequency $\omega$ equal to unity. It shows white areas which correspond 
to couples $\left[\Omega_{a} ; \Omega_{b}^{2}\right]$ not complying with the condition (32). Setting the dimensionless groups $\Omega_{a}$ and $\Omega_{b}$ to zero provides identical and unit wave numbers $(a=b=1)$. It corresponds to the E-B beam theory. In the Timoshenko beam theory, these groups are however non-zero. Considering the specimens without rubber described by Mordant (2016) and the mechanical properties as recommended in the Eurocodes, namely $E=$ $1000 f_{k}$ and $G^{*}=0.4 E$, we obtain

Figure 4 Influence of $\Omega_{a}$ and $\Omega_{b}$ on wave numbers $a$ and $b$ (or $\tilde{b}$ ) (see online version for colours)

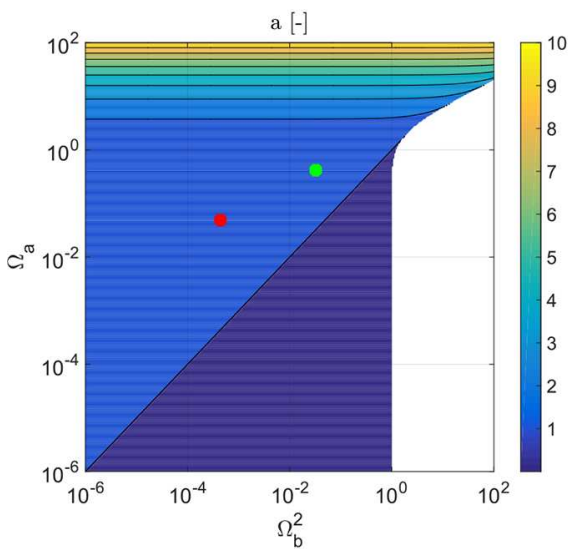

(a)

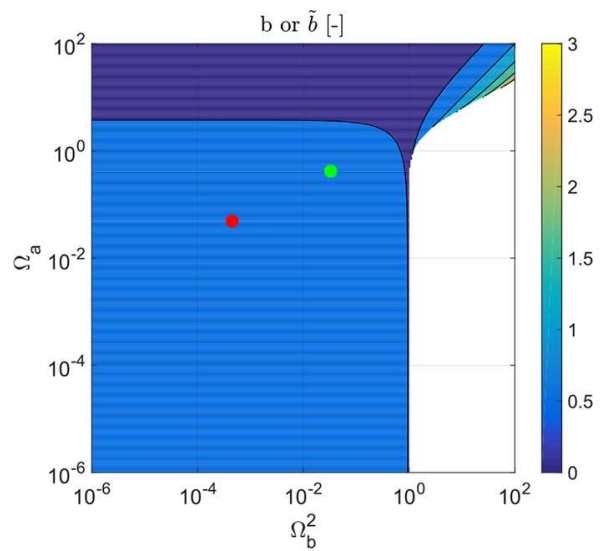

(b)

$$
\Omega_{a}=0.0489 ; \quad \Omega_{b}^{2}=4.4819 \times 10^{-4}
$$

for the short wall and

$$
\Omega_{a}=0.4159 \quad ; \quad \Omega_{b}^{2}=0.0324
$$

for the long one. These couples of values are represented in Figure 4 with red and green points, respectively. The wave numbers are $a=0.9888$ and $b=0.9885$ for the short wall, while the wave numbers for the long wall are $a=0.8901$ and $b=0.8708$. Consequently, an E-B model is sufficient to model the short wall, whereas a Timoshenko beam is required for the long wall.

\subsection{Parameters related to the additional upper mass}

Figure 5 plots the dimensionless frequency equation of the first model considering the material and geometrical properties of the short wall given in Mordant (2016). The values of the parameters related to the mass are also derived from the quantities given in this reference. The mechanical properties of the masonry are chosen according to the Eurocodes recommendations. These choices result in $\Omega_{a}=0.0489$ and $\Omega_{b}^{2}=4.4819 \times 10^{-4}$. Several variations around this nominal configuration are represented:

i no additional mass (yellow) 
ii an upper mass without gap $H_{b}$ between the beam end and the centroid of the mass (green)

iii an additional mass without rotary inertia $I_{\text {cin }}$ (red)

iv a combination of (ii) and (iii) (black).

Figure 5 Frequency equation with different assumptions (first model) - short wall without rubber (see online version for colours)

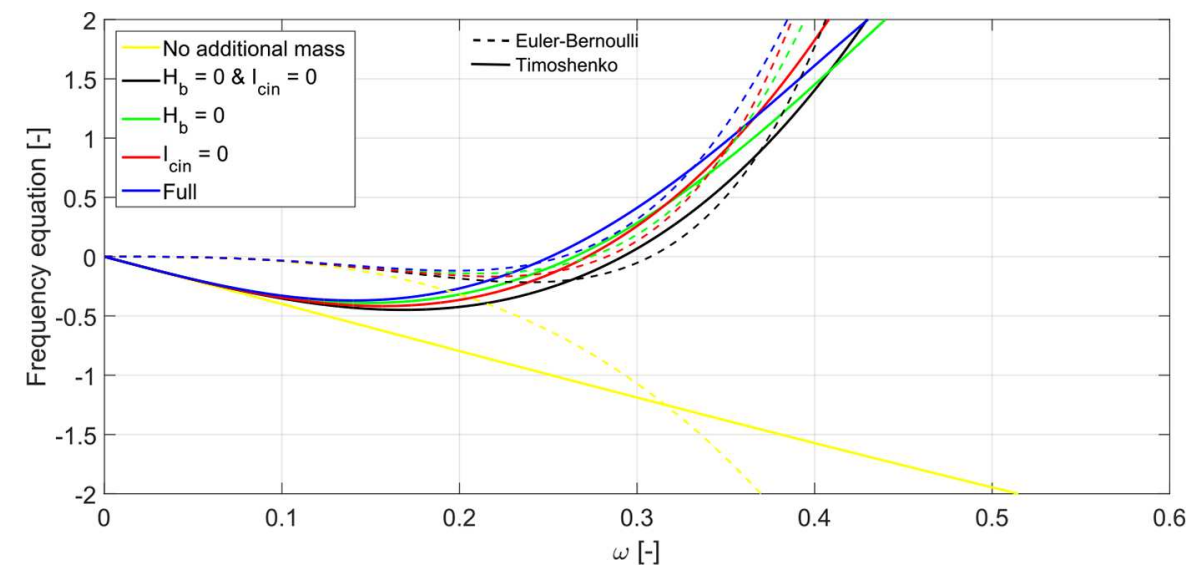

Figure 6 gives the same results in the case of the long wall, whose nominal configuration is characterised by $\Omega_{a}=0.4159$ and $\Omega_{b}^{2}=0.0324$. Beside the solid curves representing the Timoshenko model, dashed lines with similar colours represent the frequency equations obtained by the E-B beam theory (i.e. enforcing $\Omega_{a}=\Omega_{b}^{2}=0$ ) under the same assumptions.

Comparison of the different curves in Figures 5 and 6 shows the importance of the additional mass. This latter indeed provides more than $90 \%$ of the total mass and involves

Figure 6 Frequency equation with different assumptions (first model) - long wall without rubber (see online version for colours)

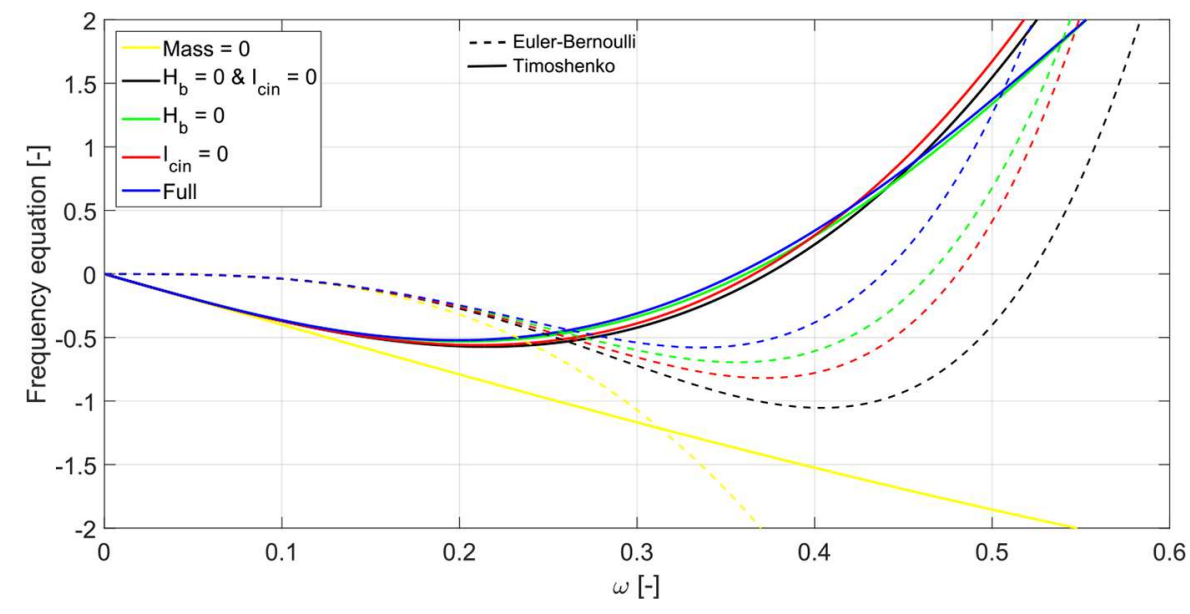


a large decrease of the first circular frequency of the beam. The influence of the gap $H_{b}$ and the rotary inertia $I_{\text {cin }}$ is more important for the short wall than for the long one. Numerical values are given in Table 1. The variation is effectively higher for the short wall (from 5.3 to $16.2 \%$ ), whereas it is less significant for the long one (less than $4.9 \%$ ). Therefore, the contribution of the rotary inertia and the distance between the top of the wall and the centroid of the mass has to be considered, especially for the short wall.

Figures 5 and 6 also compare the frequency equations developed when considering E-B (dashed lines) and Timoshenko (solid lines) beam theories. The fundamental circular frequencies for both theories are also tabulated in Table 1. Under the assumptions (ii)-(iv), the relative difference between both theories is lower than $5 \%$ regarding the short wall. This difference ranges between 19 and $30 \%$ in the case of the long wall. These values show that the E-B model can be sufficient for the short wall, but that the Timoshenko model is necessary for the long wall, as already observed from the graphical representations. The E$\mathrm{B}$ theory with assumption (i) leads to the same result for both walls. This comes from the non-dimensional formalism defined in Section 2.

Table 1 Fundamental circular frequency with different assumptions (first model) - numerical values

\begin{tabular}{lccccc}
\hline Wall & Full & $m=0$ & $H_{b}=0$ & $I_{\text {cin }}=0$ & $H_{b}=I_{\text {cin }}=0$ \\
\cline { 2 - 5 } & & (i) & (ii) & (iii) & (iv) \\
\hline Short (Timoshenko) & 0.2513 & 3.1820 & 0.2648 & 0.2718 & 0.2918 \\
Short (Euler-Bernoulli) & 0.2579 & 3.5160 & 0.2737 & 0.2829 & 0.3075 \\
Long (Timoshenko) & 0.3560 & 2.1254 & 0.3614 & 0.3660 & 0.3735 \\
Long (Euler-Bernoulli) & 0.4383 & 3.5160 & 0.4649 & 0.4803 & 0.5215 \\
\hline
\end{tabular}

Regarding the frequency equation obtained with the Timoshenko theory, further studies on the influence of the different parameters related to the mass have been performed. The results are given in Figure 7. The influence of the magnitude of the additional mass (top), of the gap $H_{b}$ (middle) and of the rotary inertia $I_{\text {cin }}$ (bottom) are given for the short (in blue) and long (in red) walls. The results are in accordance with the values tabulated in Table 1. For example, values obtained under the assumption of no additional mass (i) or $H_{b}=0$ (ii) correspond to the intersection of the curves in Figure 7 (top and middle) with the $x$-axis. Figure 7 (bottom) provides the tabulated values with $I_{c i n}=0$ (assumption (iii)) when the ordinate is equal to -1 . Again, Figure 7 highlights the higher importance of the parameters in the case of the short wall. Additional observations can be made: first, the first circular frequency is very sensitive to variations of the additional mass. The interpretation of these results has to be carefully done because the specimen total mass varies and any increment of mass has also consequences on the rotary inertia. Second, the rotary inertia $I_{\text {cin }}$ seems to have the lowest influence on the frequency equation, especially for the long wall. 
Figure 7 Influence of the parameters related to the mass: (a) Influence of the additional mass at the top, (b) influence of the distance between the top of the wall and the centroid of the mass, (c) influence of the rotary inertia of the mass (see online version for colours)

(a)

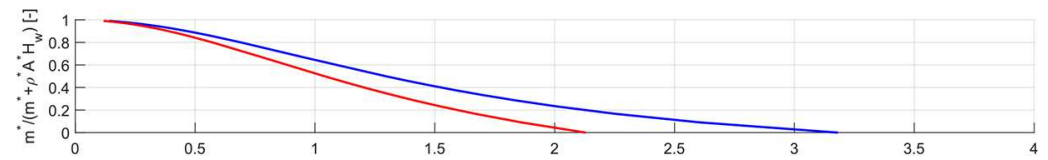

(b)

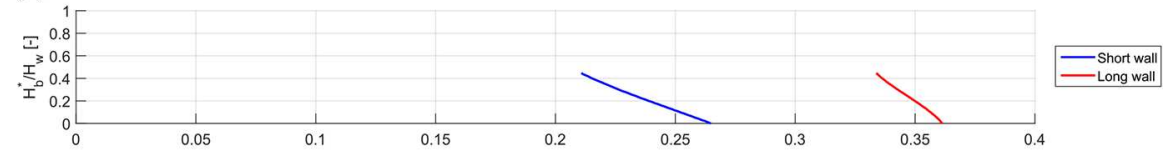

(c)

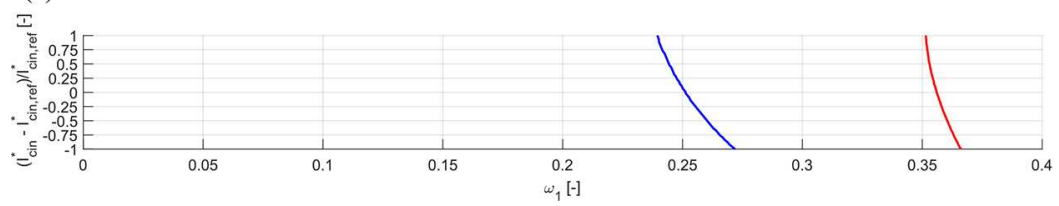

\subsection{Parameters related to the stiffness of the base and of the mass-beam connection}

The influence and importance of the presence of rubber layers leading to a beam with an elastically restrained bottom end and an additional mass elastically connected to the top is studied by comparing the frequency equation obtained with the assumption of

i a cantilever beam with an additional mass rigidly connected to its free end (first model case)

ii the same beam with an elastically restrained base

iii the same beam with a mass elastically connected to the top

iv the beam combining the effects of (ii) and (iii).

The material and geometrical properties are those of the short and long walls given by Mordant (2016). The parameters related to the additional mass are based on the quantities given in the same reference. The couple of mechanical characteristic of the rubber $\left(E_{\text {rubber }}^{*}, G_{\text {rubber }}^{*}\right)$ are chosen in the range of usual values and but are the same for the bottom and top ends. The frequency equations are re-normalised with the same circular frequency than the first model, since the beam is the same.

The results are given in Figure 8 (short wall) and Figure 9 (long wall) and the comparison is summarised in Table 2 with several observations.

The effect of the rubber layers on the natural frequency is more or less the same for both walls with a decrease in the fundamental circular frequency around $75 \%$. This conclusion is mitigated when there is only the elastically connected mass (column 3) given that the decrease is about $45 \%$ for the short wall and $63 \%$ for the long wall. Both rubber layers influence the results, even if placing a rubber layer at the bottom end only (column 4) leads to a larger decrease. Indeed, Table 2 highlights that adding the soundproofing layer between the top of the wall and the additional mass in the presence of a layer located at the 
Figure 8 Influence of the stiffness of the base and stiffness of the mass-to-wall connection (second model) - short wall with rubber (see online version for colours)

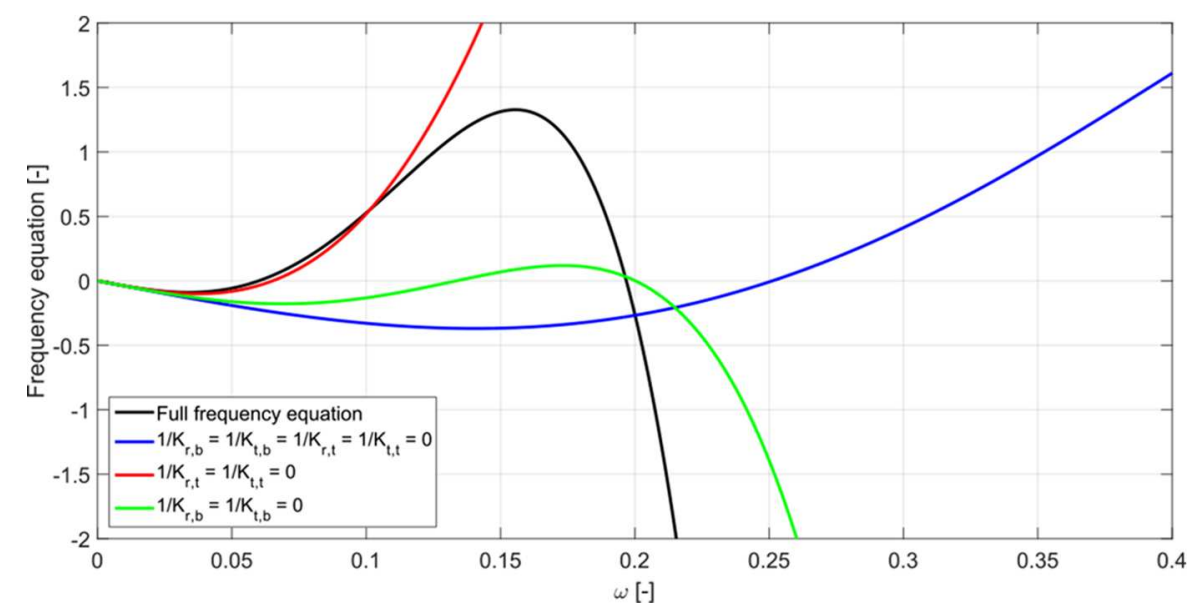

base of the wall does not involve a significant drop. Therefore, it can be concluded that the bottom rubber layer has more influence on the frequency drop. The decreases produced by the two rubber layers are not additive.

Figure 9 Influence of the stiffness of the base and stiffness of the mass-to-wall connection (second model) - long wall with rubber (see online version for colours)

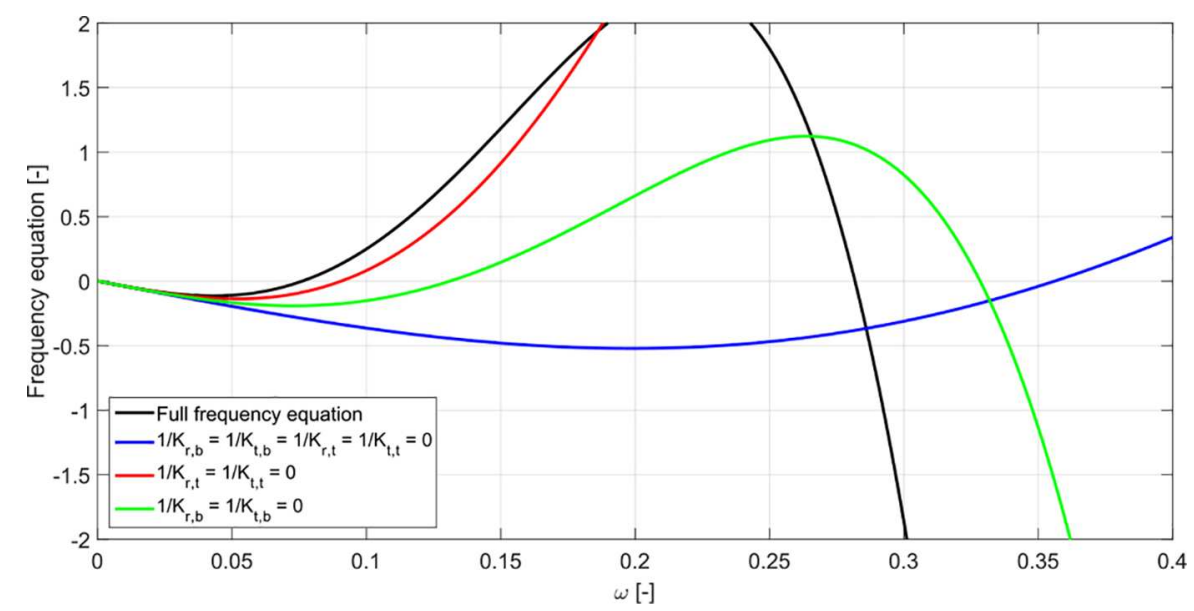

Table 2 Fundamental circular frequency with different assumptions (second model) numerical value

\begin{tabular}{lcccc}
\hline Wall & Without rubber & With rubber & $1 / K_{r, b}=1 / K_{t, b}=0$ & $1 / K_{r, t}=1 / K_{t, t}=0$ \\
\hline Short & 0.2513 & 0.0600 & 0.1336 & 0.0652 \\
Long & 0.3561 & 0.0761 & 0.1311 & 0.0906 \\
\hline
\end{tabular}




\subsection{Mode shapes}

The roots of the frequency equation provide the natural frequencies of the model. They are associated with coefficients $C_{1}, C_{2}, C_{3}$ and $C_{4}$ (respectively $\tilde{C}_{1}, \cdots, \tilde{C}_{4}$, if $\omega \Omega_{b} \geq 1$ ) and, thereby, the mode shapes $\phi(x)$ and $\psi(x)$.

The mode shapes related to the first circular frequency for each of the four tested specimens are represented in terms of displacements $\phi(x)$ (Figure 10 top) and rotations $\psi(x)$ (Figure 10 bottom). These are normalised in such a way that the maximum displacement (rotation) is equal to unity. Differences in terms of displacements between the walls without rubber devices are first observed. The mode shape for the short wall presents a vertical tangent at the base, while the mode shape of the long wall can be well approximated by a straight line. The shapes in terms of rotations are similar in each case. Such results are in accordance with previous conclusions, where the short wall could be considered as a Euler-Bernoulli beam and the shear effect are more significant for the long wall, requiring therefore a Timoshenko model. A comparison is then made between walls with the same geometry, but including or not soundproofing rubber elements. The presence of these latter involves non-zero displacement and rotation at the bottom of the wall. In particular, the rotation is mainly concentrated at the bottom of the beam, and more or less constant along the wall. The displacement mode shapes for the short and long walls are close to a straight line in the presence of rubber devices. It is therefore important not to directly conclude to the need of a Timoshenko beam model when a straight displacement mode shape is observed (e.g. short wall), as it may simply result from the compliance with the end connections.

Figure 10 Modal shapes (top : displacements - bottom : rotations) (see online version for colours)
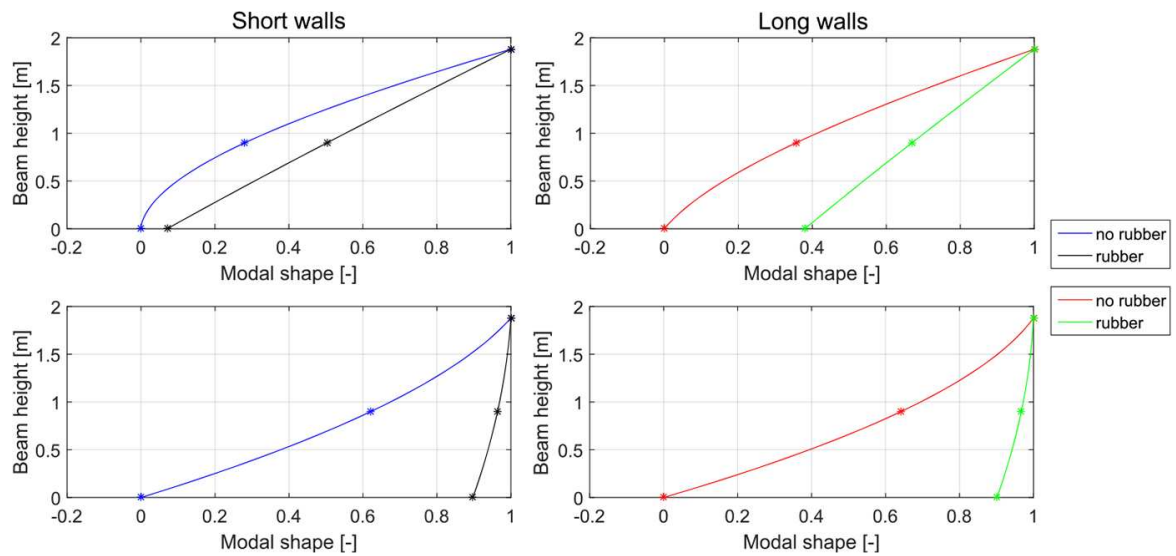

\section{Determination of equivalent mechanical properties of specific structural elements}

The expression of the frequency equation of the Timoshenko beam model depends on the two dimensionless groups $\Omega_{a}$ and $\Omega_{b}^{2}$, i.e. the ratio of the gyradius of the cross section, the beam length and the elastic-to-shear moduli ratio. These groups are explicit 
in the governing equations and implicit in the waves numbers $a, b$ and $\tilde{b}$. In the present contribution, other parameters also play an important role in the model, like the mass and the geometry of the additional mass $\left(m, H_{b}\right.$ and $\left.I_{\text {cin }}\right)$ or stiffness's involved in the boundary conditions $K_{r, b}, K_{t, b}, K_{r, t}$ and $K_{t, t}$. The determination of some of these parameters is straightforward, especially for geometrical ones. Regarding URM structures, others are more difficult to assess because of the heterogeneity of the material (units + mortar) and the large range of masonry types (clay or concrete units with mortar, glued or empty joints, etc.).

In the perspective of modelling the URM structural elements with equivalent homogeneous and isotropic beam elements at a macro-scale (equivalent frame model), the use of the frequency equation to determine equivalent mechanical properties becomes relevant. Indeed, the establishment of this equation is based on a beam model with assumptions on the material similar to what is used in equivalent beam models. Usual possible ways to estimate the mechanical properties of such elements are the execution of tests according, for instance, to EN 1052-1 or the use of empirical formulae. These latter are however derived from experimental tests and cannot cope with the specificities of every type of masonry. In the experimental campaign used as main reference for the present contribution, tests on wallets according to EN 1052-1 have been carried out prior to the dynamic testing by the producer of the masonry units to evaluate the strength of the masonry but however without including the characterisation of the elastic modulus.

In the following, the frequency equations are used to determine the fundamental frequency of URM walls without soundproofing devices prior to the shakes. This frequency is equal to $9.33 \mathrm{~Hz}$ and $4.02 \mathrm{~Hz}$ for the long and short walls respectively. Due to the lack of characterisation of the elastic and shear moduli of the masonry, recommendations of the Eurocodes are first considered. Then, a parametric study is carried out in order to define the couples of equivalent mechanical properties leading to the undamaged fundamental frequency obtained by the experiments. Finally, the more relevant couple $\left(E, G^{*}\right)$ is used as an input for the frequency equation related to walls including rubber and a parametric study is developed to identify equivalent mechanical properties of the rubber.

\subsection{Fundamental frequency using standard recommendations}

EN-1996-1-1 advises that (Section 3.7.2.(2)) "In the absence of a value determined by tests in accordance with EN 1052-1, the short-term secant modulus of elasticity of masonry, $E$, for the use in structural analysis, may be taken to be $K_{E} f_{k}$ " and recommends the value of 1000 for $K_{E}$. Notice that the value of $K_{E}$ is not divided by 2 to take into account cracking, as suggested by EN 1998-1 for seismic analysis of URM structures, because the used experimental results correspond to an undamaged configuration. EN 1996-1-1 also defines the shear modulus $G^{*}$ as $40 \%$ of the elastic modulus $E$ (Section 3.7.3.(1)). It follows,

$$
E=3900 \mathrm{MPa} ; \quad G^{*}=1560 \mathrm{MPa} .
$$

Substituting these values in the frequency equation corresponding to the simple URM walls provides the fundamental frequencies tabulated in Table 3. Comparing these results with the values obtained from the experiments shows a relative difference of 54.71 and $20.24 \%$ for the long and short walls, respectively. The recommendations of the Eurocodes lead therefore to an overestimation of the mechanical parameters in the case of URM with thin 
bed-layered joints using a tongue-and-groove system for the head joints. The discrepancy can either come from the assessment of the elastic modulus or from the ratio between the shear and elastic moduli $G^{*} / E$. In the case of masonry with empty vertical joints, the definition of a unique ratio for the estimation of the shear modulus independently from the length of the wall is quite surprising. Indeed, the number of joints increases with the length of the wall and a higher deformability in shear could be expected.

Table 3 Fundamental frequency of URM walls without rubber using the standards recommendations

\begin{tabular}{lcc}
\hline & Long wall & Short wall \\
\hline Frequency $[\mathrm{Hz}]$ & 20.82 & 5.04 \\
Dimensionless frequency [-] & 0.057 & 0.040 \\
\hline
\end{tabular}

\subsection{Characterisation of the masonry mechanical properties}

Based on the observations in the previous section, a parametric study is performed with the aim of identifying the couples $\left(E, G^{*}\right)$ that should be used in the frequency equation in order to recover the eigenfrequencies measured experimentally. Instead of adjusting $E$ and $G^{*}$ separately, we take the advantage of the fact that the ratio $G^{*} / E$ usually lies in a specific range. We will therefore identify two parameters, namely the elastic modulus $E$ and the ratio $G^{*} / E$ by constraining this identification problem to a unique Young's modulus for both long and short walls. This constraint stems from the fact that walls are built using the same masonry. The vertical empty joints should thus not influence the bending behaviour and, consequently, the value of the equivalent elastic modulus.

Figure 11 plots the results of this analysis for the long and short walls without rubber. The blue and red lines are the lieu of the pairs $\left(E, G^{*} / E\right)$ providing, with the Timoshenko beam model, the same fundamental frequency as that identified from the experiments for the long and short walls, respectively.

Figure 11 Characterisation of the masonry mechanical properties

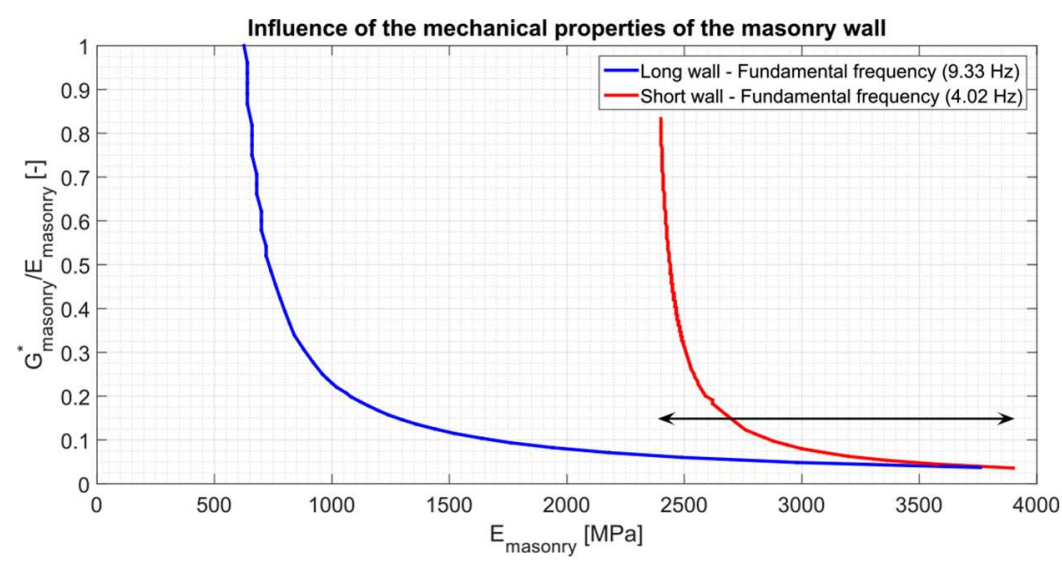


Comparison of the curves in Figure 11 highlights a higher dependency to the $G^{*} / E$ ratio of the long wall because its curve shows a more important increase in the elastic modulus when $G^{*} / E$ ratio decreases, at least for $E \gtrsim 800 \mathrm{MPa}$. Such a sensitivity is intuitively expected, when considering the ratio between the bending and shear deformabilities of the walls. Indeed, the influence of the latter becomes as important as the former in the case of the long wal, since they are of the same order of magnitude (Table 4).

This statement is further emphasised in Figures 12 and 13. Figure 12 effectively illustrates the high dependency to the $G^{*} / E$ ratio of the results for the long wall, since the curves differ while they are clearly similar for the short wall (Figure 13).

Table 4

Bending and shear deformabilities of masonry walls

\begin{tabular}{lcc}
\hline & Bending deformability $[\mathrm{m} / \mathrm{N}]$ & Shear deformability $[\mathrm{m} / \mathrm{N}]$ \\
\hline Long wall & $9.360710^{-9}$ & $9.555710^{-9}$ \\
Short wall & $2.322610^{-7}$ & $2.787110^{-8}$ \\
\hline
\end{tabular}

Figure 12 Dependence to the shear deformability (long wall) (see online version for colours)

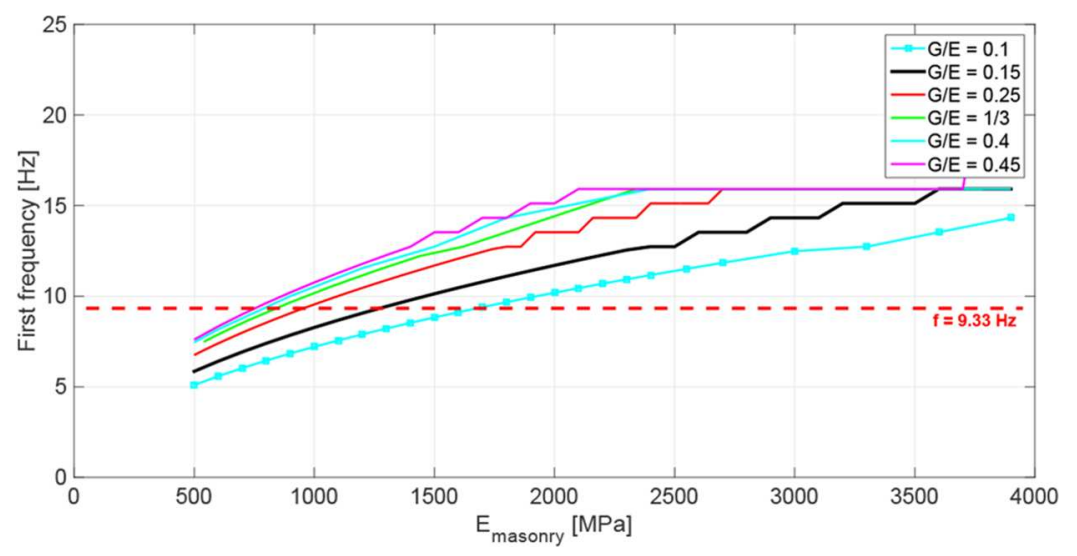

Considering the constraint on the equivalent Young's modulus, this one is identified as ranging from 2400 to $4000 \mathrm{MPa}$ (see black arrow in Figure 11). For the short wall, it is most likely around $2450 \mathrm{MPa}$ which agrees with a $G^{*} / E$ ratio between 1 and $1 / 3$. For the long wall, this leads to $G^{*} / E$ ratio lower than 0.1 . The considered limits on the interval $[1 / 3 ; 1]$ for the $G^{*} / E$ ratio correspond to the classical limitation of the Poisson's ratio. This ratio relates the elastic and shear moduli and can vary from -1 to 0.5 for a continuous material. However, such assumption is questionable in the case of masonry with empty vertical joints and the $G^{*} / E$ ratio may become lower than $1 / 3$ (Poisson's ratio virtually larger than 0.5). This range of values is also observable for other materials (Lee and Lakes, 1997). Moreover, contrary to the bending deformability, the shear deformability might suffer from size effects (i.e. number of vertical open joints), which are not accounted for 
Figure 13 Dependence to the shear deformability (short wall) (see online version for colours)

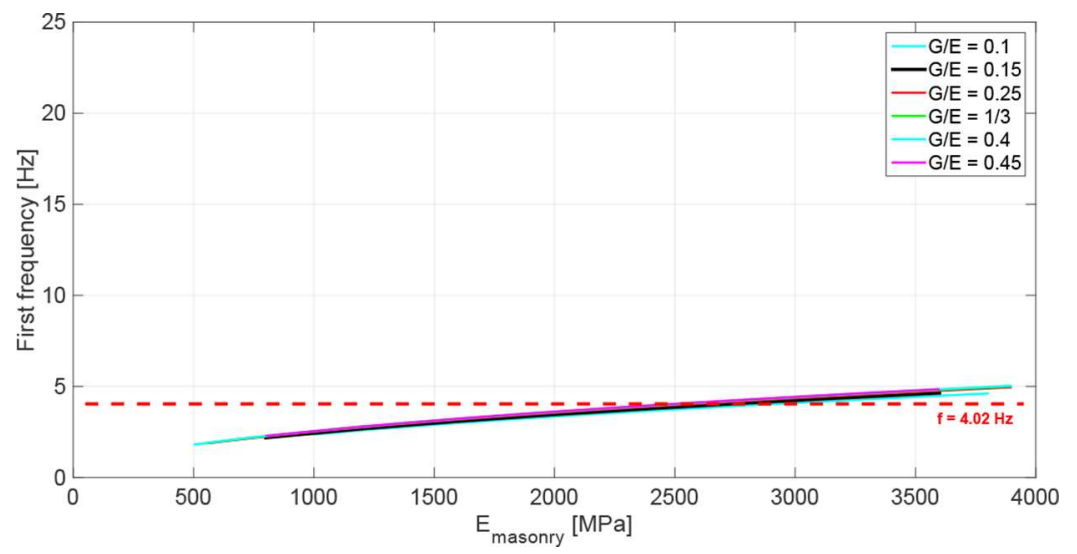

in our homogenised Timoshenko beam model, resulting therefore in an equivalent shear modulus lower for the long wall.

The recommended ratio of 40\% given by EN 1996-1-1 appears to be relevant for walls with a few vertical joints, as for example the short wall. The corresponding equivalent elastic modulus is about $2465 \mathrm{MPa}$, which is $K_{E}=632$ times the characteristic compressive strength. For longer walls, the ratio $G^{*} / E$ has to be reduced by $6.25 \%$ to take into account the extra vertical gaps between the units. A lower limit should exist, but its determination requires more experimental investigations. These conclusions are supported by the results found by Gregoire (2007) or da Porto et al. (2009). In the latter, uniaxial and diagonal compression tests were carried out on specimens with average dimensions of $1000 \times 1000 \times 300 \mathrm{~mm}$. The masonry bounding was similar to the one considered in this paper. Indeed, the characteristic compressive strength (NBN-EN 1996-1-1) $f_{k}$ is 5.493 $\mathrm{MPa}$ for units of group 2. The authors give an elastic modulus of $4424 \mathrm{MPa}$, namely 805 $f_{k}$, and the ratio $G^{*} / E$ is equal to $17.02 \%$. Another experimental campaign on the same type of masonry tested in static-cyclic conditions led to the same conclusions (Mordant, 2016). In particular, the equivalent elastic modulus found in this campaign is similar to the values considered here.

\subsection{Characterisation of the rubber mechanical properties}

The previous sections deal with the characterisation of the masonry mechanical properties and the comparison with current standard recommendations. It results in the determination of acceptable couples of elastic and shear moduli for the first model, leading to the natural frequency measured experimentally. Among the possible combinations, two will be chosen as references for the short and long walls, respectively

$$
\begin{aligned}
& E=2465 \mathrm{MPa} \quad ; \quad G^{*} / E=0.4 \quad \text { (short wall) } \\
& E=2465 \mathrm{MPa} \quad ; \quad G^{*} / E=0.0625 \quad \text { (long wall). }
\end{aligned}
$$

Once the masonry mechanical properties are determined and the geometry is clearly defined, the expression of the frequency equation of the second model has only two 
unknowns left, namely the mechanical properties of the rubber layers, placed at the base of the wall and between the top of the wall and the additional mass. These properties are translated via the values of the stiffnesses $K_{r, b}, K_{t, b}, K_{r, t}$ and $K_{t, t}$ present in the frequency equation.

The results presented in Figure 14 provide the coupled values ( $E_{\text {rubber }}^{*}, G_{\text {rubber }}^{*}$ ) $E_{\text {rubber }}^{*}$ ) giving the first natural frequency measured experimentally. The mechanical properties of the rubber layers are the same for the top and bottom as the same material is used. Contrary to the masonry wall, the rubber layers can be considered as a homogeneous and continuous body. There is therefore a relation between the elastic and shear moduli through Poisson ratio

$$
G_{\text {rubber }}^{*}=\frac{E_{\text {rubber }}^{*}}{2\left(1+\nu_{\text {rubber }}\right)} .
$$

For rubber elements, the Poisson's ratio is close to the incompressibility limit $\nu=0.5$ and leads to a $G_{\text {rubber }}^{*} / E_{\text {rubber }}^{*}$ ratio of $1 / 3$. The corresponding elastic modulus is $11.1 \mathrm{MPa}$ for the short wall and 7.5 MPa for the long one. The long wall also exhibits a greater dependency on the Poisson's ratio in comparison to short wall (see Figure 14).

Figure 14 Characterisation of the mechanical properties of the rubber layers

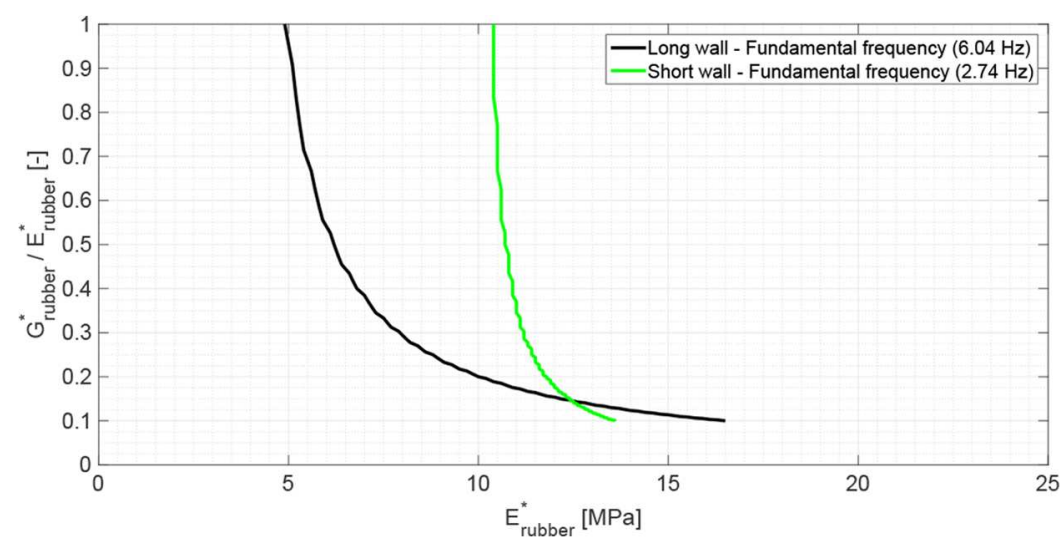

These results are in accordance with the experimental measurements and the producer's prescriptions (Mordant, 2016). The difference between the identified values (11.1 and 7.5 $\mathrm{MPa}$ ) has several causes. On the one hand, the rubber layer works under different compressive stresses for the short and long walls. It is well known that the rubber exhibits a lower deformability under a larger stress, hence the rubber placed at the extremities of the short wall has a larger elastic modulus. On the other hand, the mechanical properties of the rubber devices show some significant variability due to their manufacturing process, i.e. they are made of recycled rubber, involving a possible scatter in the properties.

\section{Conclusion}

This paper is dedicated to the development of the frequency equation of a modified cantilever beam, using the Timoshenko beam theory. Two models are studied to consider 
the modifications of the classic cantilever beam. The first model consists in a classical cantilever beam with an additional mass rigidly connected to the free end. The second one includes an elastically restrained base and a flexible connection between the free end and the additional mass. These modifications are taken into account through a proper modification of the boundary conditions. The deduced frequency equations highlight the importance the dimensionless groups $\Omega_{a}$ and $\Omega_{b}^{2}$ which include the geometrical and mechanical parameters of the beam as well as the stiffness of the base and mass-to-beam connections. Then, the frequency equations are used to characterise equivalent mechanical properties of load-bearing clay URM walls including soundproofing devices. Tested on a shaking table, these walls have glued horizontal joints and empty vertical ones. Rubber layers are placed at their base and top for acoustic performance reasons. One of the main outcomes of the experimental tests is the assessment of the natural frequencies of the walls, based on the measurements of accelerations during white noise identification tests. For the first model, the characterisation leads to the definition of combined values for equivalent elastic and shear moduli of the masonry walls, $E$ and $G^{*}$, expressed by the combination of the elastic modulus $E$ and the $G^{*} / E$ ratio. The second model is focused on the mechanical properties of the soundproofing devices. Finally, a comparison of the results in terms of mechanical properties with current standard recommendations for masonry structures shows an overestimation of the recommended values for the considered type of masonry. This paper however proves that the modelling of masonry walls thanks to equivalent beam using the Timoshenko beam theory is possible.

\section{Acknowledgements}

The research leading to these results has received funding from the European Union Seventh Framework Program (FP7/2007-2013) under grant agreement $\mathrm{n}^{\circ} 227887$, SERIES. The authors acknowledge the support received from the EQUALS laboratory and the University of Bristol for the access to their facilities and their help during the experimental campaign. C. Mordant also acknowledges the direct support received from F.R.I.A. (F.R.S.-FNRS - Belgian Fund for Scientific Research).

\section{References}

Ansari, M., Jalili, N. and Esmailzadeh, E. (2011) 'Exact frequency analysis of a rotating cantilever beam with tip mass subjected to torsional-bending vibrations', Journal of Vibration and Acoustics, Vol. 133, No. 4, p.041003.

Braga, F. and Liberatore D. (1990) 'A finite element for the analysis of the response of masonry buildings', Proceedings of 5th North American masonry conference. University of Illinois at Urbana-Champaign, 3-6 June.

Bruch Jr, J.C. and Mitchell, T.P. (1987) 'Vibrations of a mass-loaded clamped-free Timoshenko beam', Journal of Sound and Vibration, Vol. 114, No. 2, pp.341-345.

Calio, I. and Greco, A. (2012) 'Free vibrations of Timoshenko beam-columns on Pasternak foundations', Journal of Vibration and Control, Vol. 19, No. 5, pp.686-696. doi:10.1177/ 1077546311433609. http://jvc.sagepub.com/cgi/doi/10.1177/1077546311433609

da Porto, F., Grendene, M. and Modena, C. (2009) 'Estimation of load reduction factors for clay masonry walls', Earthquake Engineering \& Structural Dynamics, Vol. 38, No. 10, pp.1155-1174. doi:10.1002/eqe.887. http://doi.wiley.com/10.1002/eqe.887 
Esmailzadeh, E. and Ohadi, A.R. (2000) 'Vibration and stability analysis of non-uniform Timoshenko beams under axial and distributed tangential loads', Journal of Sound and Vibration, Vol. 236, No. 3, pp.443-456. doi:10.1006/jsvi.2000.2999. http://linkinghub.elsevier. com/retrieve/pii/S0022460X00929997

Farchaly, S. and Shebl, M. (1995) 'Exact frequency and mode shape formulae for studying vibration and stability of Timoshenko beam system', Journal of Sound and Vibration, Vol. 180, pp.205-227. http://www.sciencedirect.com/science/article/pii/S0022460X85700752

Gregoire, Y. (2007) 'Compressive strength of masonry according to eurocode 6: a contribution to the study of the influence of shape factors', Masonry International, Vol. 20, No. 2, p.69.

Han, S., Benaroya, H. and Wei, T. (1999) 'Dynamics of transversely vibrating beams using four engineering theories', Journal of Sound and Vibration, Vol. 225, pp.935-988. http://www.sciencedirect.com/science/article/pii/S0022460X99922575

Hernández-Urrea, J. and Dario Aristizábal-Ochoa, J. (2008) 'Static and dynamic stability of an elastically restrained Beck column with an attached end mass', Journal of Sound and Vibration, Vol. 312, Nos. 4-5, pp.789-800. doi:10.1016/j.jsv.2007.11.014. http://linkinghub.elsevier.com/ retrieve/pii/S0022460X0700908X

Lagomarsino, S., Penna, A., Galasco, A. and Cattari, S. (2013) 'Tremuri program: an equivalent frame model for the nonlinear seismic analysis of masonry buildings', Engineering Structures, Vol. 56, pp.1787-1799.

Lee, T. and Lakes, R. (1997) 'Anisotropic polyurethane foam with poisson'sratio greater than 1', Journal of Materials Science, Vol. 32, No. 9, pp.2397-2401.

Low, K. (1999) 'Comparisons of experimental and numerical frequencies for classical beams carrying a mass in-span', International Journal of Mechanical Sciences, Vol. 41, No. 12, pp.1515-1531. doi:10.1016/S0020-7403(98)00103-9. http://linkinghub.elsevier.com/ retrieve/pii/S0020740398001039

Low, K.H. (2000) 'A modified Dunkerley formula for eigenfrequencies of beams carrying concentrated masses', International Journal of Mechanical Sciences, Vol. 42, pp.1287-1305.

Li, X. (2013) 'Free vibration of axially loaded shear beams carrying elastically restrained lumpedtip masses via asymptotic timoshenko beam theory', Journal of Engineering Mechanics, Vol. 139, No. 4, pp.418-428. doi:10.1061/(ASCE)EM.1943-7889.0000403. http://ascelibrary. org/doi/full/10.1061/(ASCE)EM.1943-7889.0000403

Li, X-F., Kang, Y-A. and Wu, J-X. (2013a) 'Exact frequency equations of free vibration of exponentially functionally graded beams', Applied Acoustics, Vol. 74, No. 3, pp.413-420. doi:10.1016/j.apacoust.2012.08.003. http://linkinghub.elsevier.com/retrieve/pii/ S0003682X12002538

Kappos, A.J., Penelis, G.G. and Drakopoulos, C.G. (2002) 'Evaluation of simplified models for lateral load analysis of unreinforced masonry buildings', Journal of structural Engineering, Vol. 128, No. 7, pp.890-897.

Khaji, N., Shafiei, M. and Jalalpour, M. (2009) 'Closed-form solutions for crack detection problem of Timoshenko beams with various boundary conditions', International Journal of Mechanical Sciences, Vol. 51, Nos. 9-10, pp.667-681. doi:10.1016/j.ijmecsci.2009.07.004. http://inkinghub.elsevier.com/retrieve/pii/S0020740309001398

Majkut, L. (2009) 'Free and forced vibrations of timoshenko beams described by single difference equation', Journal of Theoretical and Applied Mechanics, Vol. 47, No. 1, pp.193-210. http://yadda.icm.edu.pl/baztech/element/bwmeta1.element.baztech-article-BWM4-0019-0012

Mordant, C. (2016) Unreinforced Clay Masonry Structures: Advanced Characterisation of the Seismic Behaviour Including Acoustic Issues, Doctoral Dissertation, University of Liège and Hasselt Uniersity.

Mordant, C., Dietz, M.S., Taylor, C.A., Plumier, A. and Degée, H. (2014) 'Seismic behavior of thinbed layered unreinforced clay masonry shear walls including soundproofing elements', Seismic Evaluation and Rehabilitation of Structures, Springer, Berlin, pp.77-93.

Naguleswaran, S. (2003) 'Vibration and stability of an Euler-Bernoulli beam with up to threestep changes in cross-section and in axial force', International Journal of Mechanical 
Sciences, Vol. 45, No. 9, pp.1563-1579. doi:10.1016/j.ijmecsci.2003.09.001. http://linkinghub. elsevier.com/retrieve/pii/S0020740303001577

Oguamanam, D. (2003) 'Free vibration of beams with finite mass rigid tip load and flexuraltorsional coupling', International Journal of Mechanical Sciences, Vol. 45, Nos. 6-7, pp.963-979. doi:10.1016/j.ijmecsci.2003.09.014.

Penna, A., Lagomarsino, S. and Galasco, A. (2013) 'A nonlinear macroelement model for the seismic analysis of masonry buildings', Earthquake Engineering \& Structural Dynamics, Vol. 43, No. 2, pp.159-179. doi:10.1002/eqe.2335. http://doi.wiley.com/10.1002/eqe.2335

Salarieh, H. and Ghorashi, M. (2006) 'Free vibration of Timoshenko beam with finite mass rigid tip load and flexural-torsional coupling', International Journal of Mechanical Sciences, Vol. 48, No. 7, pp.763-779. doi:10.1016/j.ijmecsci.2006.01.008. http://linkinghub.elsevier.com/ retrieve/pii/S0020740306000269

Strutt, J.W. (1877) The theory of sound, London, Macmillan and co, p.370.

Timoshenko, S.P. (1921) 'On the correction for shear of the differential equation for transverse vibrations of bars of uniform cross-section', Philosophical Magazine, Series 6, Vol. 41, pp.744-746.

Timoshenko, S.P. (1922) 'X. On the transverse vibrations of bars of uniform cross-section', The London, Edinburgh, and Dublin Philosophical Magazine and Journal of Science, Vol. 43, No. 253, pp.125-131.

Timoshenko, S.P. and Goodier, J.N. (1987) Theory of Elasticity, 3rd ed., McGraw-Hill Book Company, New-York.

Zhang, H., Kang, Y. and Li, X. (2013) 'Stability and vibration analysis of axially-loaded shear beam-columns carrying elastically restrained mass', Applied Mathematical Modelling, Vol. 37, Nos. 16-17, pp.8237-8250. doi:10.1016/j.apm.2013.03.050. http://linkinghub.elsevier.com/ retrieve/pii/S0307904X13002242 


\section{Nomenclature}

\begin{tabular}{|c|c|c|c|c|}
\hline \multicolumn{3}{|c|}{ Dimensional variables } & \multicolumn{2}{|c|}{$\begin{array}{c}\text { Corresponding } \\
\text { dimensionless variables }\end{array}$} \\
\hline$A^{*}$ & cross-sectional area & $m^{2}$ & $A$ & $=A^{*} / H_{w}^{2}$ \\
\hline $\begin{array}{c}a^{*}, b^{*}, \tilde{b}^{*} \\
E \\
f\end{array}$ & $\begin{array}{c}\text { wave number } \\
\text { elastic modulus } \\
\text { transverse force per } \\
\text { length }\end{array}$ & $\begin{array}{c}1 / m \\
N / m^{2} \\
m^{2}\end{array}$ & $a, b, \tilde{b}$ & $=a^{*} \cdot H_{w}, b^{*} \cdot H_{w}, \tilde{b}^{*} \cdot H_{w}$ \\
\hline$G^{*}$ & shear modulus & $N / m^{2}$ & $G$ & $=G^{*} \cdot H_{w}^{4} / E I^{*}$ \\
\hline$H_{w}$ & height of the beam & $m$ & & \\
\hline$H_{b}^{*}$ & lever arm & $m$ & $H_{b}$ & $=H_{b}^{*} / H_{w}$ \\
\hline$I^{*}$ & $\begin{array}{l}\text { bending inertia of the } \\
\text { beam }\end{array}$ & $m^{4}$ & $I$ & $=I^{*} / H_{w}^{4}$ \\
\hline$I_{\text {cin }}^{*}$ & $\begin{array}{l}\text { rotary inertia of the } \\
\text { additional upper mass }\end{array}$ & $\mathrm{kg} \cdot \mathrm{m}^{2}$ & $I_{\text {cin }}$ & $=I_{c i n}^{*} \cdot \omega_{1}^{* 2} \cdot H_{w} / E I^{*}$ \\
\hline$k^{\prime}$ & shape factor & - & & \\
\hline$K_{t, b}^{*}$ & $\begin{array}{c}\text { base spring translational } \\
\text { stiffness }\end{array}$ & $N / m$ & $K_{t, b}$ & $=K_{t, b}^{*} \cdot H_{w}^{3} / E I^{*}$ \\
\hline$K_{r, b}^{*}$ & $\begin{array}{c}\text { base spring rotational } \\
\text { stiffness }\end{array}$ & N.m & $K_{r, b}$ & $=K_{r, b}^{*} \cdot H_{w} / E I^{*}$ \\
\hline$K_{t, t}^{*}$ & $\begin{array}{l}\text { top spring translational } \\
\text { stiffness }\end{array}$ & $N / m$ & $K_{t, t}$ & $=K_{t, t}^{*} \cdot H_{w}^{3} / E I^{*}$ \\
\hline$K_{r, t}^{*}$ & $\begin{array}{l}\text { top spring rotational } \\
\text { stiffness }\end{array}$ & N.m & $K_{r, t}$ & $=K_{r, t}^{*} \cdot H_{w} / E I^{*}$ \\
\hline$M^{*}$ & bending moment & N.m & $M$ & $=M^{*} \cdot H_{w} / E I^{*}$ \\
\hline$m^{*}$ & $\begin{array}{l}\text { magnitude of the } \\
\text { additional upper mass }\end{array}$ & $k g$ & $m$ & $=m^{*} \cdot \omega_{1}^{* 2} \cdot H_{w}^{3} / E I^{*}$ \\
\hline$t^{*}$ & time & $s$ & $t$ & $=t^{*} \cdot \omega_{1}^{*}$ \\
\hline$v^{*}$ & $\begin{array}{c}\text { transverse displacement } \\
\text { of the beam }\end{array}$ & $m$ & $v$ & $=v^{*} / H_{w}$ \\
\hline$V^{*}$ & shear force & $N$ & $V$ & $=V * . H_{w}^{2} / E I^{*}$ \\
\hline$x^{*}$ & $\begin{array}{l}\text { axial coordinate of the } \\
\text { beam }\end{array}$ & $m$ & $x$ & $=x^{*} / H_{w}$ \\
\hline$\alpha$ & $\begin{array}{l}\text { angle of rotation of the } \\
\text { beam due to bending }\end{array}$ & rad & & \\
\hline$\rho^{*}$ & density of the beam & $\mathrm{kg} / \mathrm{m}^{3}$ & $\rho$ & $=\rho^{*} \cdot \omega_{1}^{* 2} \cdot H_{w}^{6} / E I^{*}$ \\
\hline$\omega^{*}$ & angular frequency & $\mathrm{rad} / \mathrm{s}$ & $\omega$ & $=\omega^{*} / \omega_{1}^{*}$ \\
\hline
\end{tabular}

